\title{
High-density lipoprotein cholesterol efflux capacity is not associated with atherosclerosis and prevalence of cardiovascular outcome
}

Citation for published version (APA):

Josefs, T., Wouters, K., Tietge, U. J. F., Annema, W., Dullaart, R. P. F., Vaisar, T., Arts, I. C. W., van der Kallen, C. J. H., Stehouwer, C. D. A., Schalkwijk, C. G., Goldberg, I. J., Fisher, E. A., \& van

Greevenbroek, M. M. J. (2020). High-density lipoprotein cholesterol efflux capacity is not associated with atherosclerosis and prevalence of cardiovascular outcome: The CODAM study. Journal of Clinical Lipidology, 14(1), 122-132.e4. https://doi.org/10.1016/j.jacl.2019.10.012

Document status and date:

Published: 01/01/2020

DOI:

10.1016/j.jacl.2019.10.012

Document Version:

Publisher's PDF, also known as Version of record

Document license:

Taverne

Please check the document version of this publication:

- A submitted manuscript is the version of the article upon submission and before peer-review. There can be important differences between the submitted version and the official published version of record.

People interested in the research are advised to contact the author for the final version of the publication, or visit the DOI to the publisher's website.

- The final author version and the galley proof are versions of the publication after peer review.

- The final published version features the final layout of the paper including the volume, issue and page numbers.

Link to publication

\footnotetext{
General rights rights.

- You may freely distribute the URL identifying the publication in the public portal. please follow below link for the End User Agreement:

www.umlib.nl/taverne-license

Take down policy

If you believe that this document breaches copyright please contact us at:

repository@maastrichtuniversity.nl

providing details and we will investigate your claim.
}

Copyright and moral rights for the publications made accessible in the public portal are retained by the authors and/or other copyright owners and it is a condition of accessing publications that users recognise and abide by the legal requirements associated with these

- Users may download and print one copy of any publication from the public portal for the purpose of private study or research.

- You may not further distribute the material or use it for any profit-making activity or commercial gain

If the publication is distributed under the terms of Article $25 \mathrm{fa}$ of the Dutch Copyright Act, indicated by the "Taverne" license above, 


\title{
High-density lipoprotein cholesterol efflux prevalence of cardiovascular outcome: The CODAM study
}

\author{
Tatjana Josefs, MSc, Kristiaan Wouters, PhD, Uwe J. F. Tietge, MD, PhD, \\ Wijtske Annema, PhD, Robin P. F. Dullaart, MD, PhD, Tomas Vaisar, PhD, \\ Ilja C. W. Arts, PhD, Carla J. H. van der Kallen, PhD, Coen D. A. Stehouwer, MD, PhD, \\ Casper G. Schalkwijk, PhD, Ira J. Goldberg, MD, Edward A. Fisher, MD, MPH, PhD, \\ Marleen M. J. van Greevenbroek, PhD*
}

Department of Internal Medicine and CARIM School for Cardiovascular Diseases, Maastricht University Medical Centre, Maastricht, The Netherlands (Drs Josefs, Wouters, van der Kallen, Stehouwer, Schalkwijk, and van Greevenbroek); Division of Cardiology, Department of Medicine, New York University School of Medicine, New York, New York (Drs Josefs and Fisher); Division of Clinical Chemistry, Department of Laboratory Medicine, Karolinska Institute, Stockholm, Sweden (Dr Tietge); Clinical Chemistry, Karolinska University Laboratory, Karolinska University Hospital, Stockholm, Sweden (Dr Tietge); Institute of Clinical Chemistry, University Hospital of Zurich and University of Zurich, Zurich, Switzerland (Dr Annema); Department of Endocrinology, University of Groningen, University Medical Center Groningen, The Netherlands (Dr Dullaart); Department of Medicine, UW Medicine Diabetes Institute, University of Washington, Seattle, Washington (Dr Vaisar); Maastricht Centre for Systems Biology (MaCSBio), Department of Epidemiology, and CARIM School for Cardiovascular Diseases, Maastricht University Medical Centre, Maastricht, The Netherlands (Dr Arts); and Division of Endocrinology, Diabetes and Metabolism, Department of Medicine, New York University School of Medicine, New York, New York (Dr Goldberg)

KEYWORDS:

Atherosclerosis;

Cardiovascular diseases;

High-density lipoprotein;
BACKGROUND: Cholesterol Efflux Capacity (CEC) is considered to be a key atheroprotective property of high-density lipoproteins (HDL). However, the role of HDL-CEC in atherosclerosis and cardiovascular $(\mathrm{CV})$ risk is still controversial, and data in individuals with diabetes are limited.

OBJECTIVE: In this study, we have investigated the relationship of CEC and other HDL characteristics with clinical and subclinical atherosclerosis in subjects with elevated cardiovascular diseases (CVD) risk and Type 2 Diabetes Mellitus (T2DM).
Conflict of interest: The authors declare that they have no conflict of interest.

Contribution: T.J. and M.M.J.vG performed the analyses, and T.J. created the Figures and Tables. T.J., K.W. and M.M.J.vG wrote and edited the manuscript. U.J.F.T., W.A., R.P.F.D., T.V. performed HDL-CEC assays, I.C.W.A. contributed to the lipoprotein measurements, M.M.J.vG., C.J.H.K., C.D.A.S., C.G.S. are part of the CODAM steering committee, E.A.F and I.J.G were involved in setting up the study. All co-authors read and edited the manuscript.

Sources of funding: The Netherlands Organization for Scientific Research (940-35-034; CODAM study), Dutch Diabetes Research Foundation (98.901; CODAM study). AHA Predoctoral Fellowship (18PRE33990436; TJ), NIH grants (HL129433; HL092969; 123398; EAF, IJG, TJ). Part of the measurements were supported through grants from The Maastricht University Medical Center+ and the Biobanking and Biomolecular Resources Research Infrastructure (BBMRI) Metabolomics Consortium funded by BBMRI-NL, a research infrastructure financed by the Dutch government (NWO, 184.021.007 and 184033111).

* Corresponding author. Department of Internal Medicine, Maastricht University Medical Centre, CARIM School for Cardiovascular Diseases, PO BOX 616, 6200 MD The Netherlands.

E-mail address: m.vangreevenbroek@maastrichtuniversity.nl

Submitted June 27, 2019. Accepted for publication October 23, 2019. 
Cholesterol efflux capacity
METHODS: Using multiple linear regression analyses, we determined the relationship of HDL-CEC with carotid intima-media thickness (cIMT, Z-Score), an endothelial dysfunction (EnD) Score (ZScore), prevalent CVD ( $\mathrm{n}=150$ cases) and history of CV events (CVE, $\mathrm{n}=85$ cases) in an observational cohort (CODAM, $\mathrm{n}=574,59.6 \pm 0.3 \mathrm{yr}, 61.3 \%$ men, $24.4 \%$ T2DM). Stratified analyses were performed to determine if the associations differed between individuals with normal glucose metabolism (NGM) and those with disturbed glucose metabolism.

RESULTS: HDL-CEC was not associated with either marker of atherosclerosis (cIMT, EnD Score) nor with CVD or CVE. In contrast, other HDL characteristics that is, HDL-Cholesterol (HDL-C, ZScore), apolipoprotein A-I (apoA-I, Z-Score), HDL size (Z-Score) and HDL particle number (HDL$\mathrm{P}, \mathrm{Z}$-Score) were inversely and significantly associated with the EnD Score (s -0.226 to -0.097 , $P<.05$ ) and CVE (ORs 0.61 to $0.68, P<.05$ ). In stratified analyses, HDL size and HDL-P were significantly associated with the EnD Score in individuals with NGM ( $P_{\text {interaction }} .039$ and .005 , respectively), but not in those with (pre)diabetes. HDL-C and apoA-I were inversely associated with prevalent CVD in individuals with (pre)diabetes $\left(P_{\text {interaction }}=.074\right.$ and .034 , respectively), but not in those with NGM.

CONCLUSION: HDL-CEC is not associated with clinical or subclinical atherosclerosis, neither in the whole population nor in individuals with (pre)diabetes, while other HDL characteristics show atheroprotective associations. The atheroprotective associations of HDL-size and HDL-P are lost in (pre) diabetes, while higher concentrations of HDL-C and apoA-I are associated with a lower prevalence of CVD in (pre)diabetes.

(C) 2020 National Lipid Association. All rights reserved.

\section{Introduction}

High-density Lipoprotein (HDL) is well known for its cardioprotective effect, resulting from, among others, its antiinflammatory and endothelial protective properties. The key cardioprotective effect of HDL is thought to originate in its cholesterol efflux capacity (HDL-CEC), which represents the first step in reverse cholesterol transport. ${ }^{1,2}$ Since up till now, the results of HDL Cholesterol (HDLC) raising trials have been disappointing, ${ }^{3,4}$ the importance of HDL function in cardiovascular diseases (CVD) has become a topic of major interest over the last few years.

HDL-mediated cholesterol efflux may lead to cholesterol reduction in the atherosclerotic plaque by promoting the removal of excess cholesterol from plaque macrophage foam cells and transporting it back to the liver for its excretion into the bile. ${ }^{5,6}$ So far, animal studies showed a link between macrophage-specific cholesterol efflux and prevention of atherosclerosis. ${ }^{7-10}$ Further, functional HDL was shown to promote atherosclerosis regression. ${ }^{11,12}$ However, it has also been shown that, besides antiinflammatory effects, ${ }^{13}$ cholesterol efflux can also exert proinflammatory effects on mouse and human macrophages, which are thought to be detrimental for atherosclerosis. ${ }^{14,15}$ In human studies, an inverse association between HDL-CEC and the prevalence $^{16}$ and incidence of CV events $(\mathrm{CVE})^{17-19}$ was shown, which remained present after adjustment for CV risk factors, including HDL-C, ${ }^{19}$ HDL-C and apoA-I, ${ }^{16,18}$ and HDL-C and HDL particle number (HDL-P). ${ }^{17}$ In contrast, another study showed that a higher HDL-CEC was associated with increased risk of incident CVE, also after adjustment for CV risk factors, including HDL-C and LDL-C. ${ }^{20}$ The reasons for these apparent discrepancies are currently unclear.
Diabetes increases the risk of CVD by 2 to 4 -fold. ${ }^{21}$ While the underlying mechanisms remain to be fully understood, altered levels or impaired functions of circulating lipoproteins are likely to be involved. Some studies have shown that HDL-CEC is impaired in patients with insulin resistance ${ }^{22}$ and type 2 diabetes mellitus (T2DM), ${ }^{18,23}$ but other studies did not find any difference ${ }^{24}$ or even increased HDL-CEC compared to healthy controls. ${ }^{25-29}$ While CV outcome studies demonstrated that HDL-CEC did not differ between non-T2DM and T2DM subjects, they showed that statin treatment in both groups leads to an increase in HDLCEC, which correlates with beneficial changes on plaque morphology. ${ }^{30,31}$

Taken together, the outcomes of human studies on the relationship between HDL-CEC and CVD have been controversial, and data in individuals with diabetes are limited. The goal of this study was to evaluate the associations of HDL-CEC and other characteristics of HDL on (sub)clinical atherosclerotic disease, as represented by carotid intima-media thickness (cIMT) and an endothelial dysfunction Score (EnD Score), prevalent CVD and history of CVE. In addition, we determined whether such associations differ between individuals with normal glucose metabolism (NGM) and those with prediabetes or diabetes (hereafter referred to as (pre)diabetes) and are the first to report on the relationship of CEC with CVD/CVE in individuals with (pre)diabetes. This evaluation was done in a well-established human cohort that is characterized by a moderately increased risk of cardiometabolic disease, that is, the Cohort on Diabetes and Atherosclerosis Maastricht (CODAM). We hypothesized that a higher HDL-CEC is associated with less (sub)clinical atherosclerotic disease and that this association is attenuated in individuals with (pre)diabetes. 


\section{Patients \& methods}

\section{Study population}

CODAM includes 574 participants who were selected from a large cohort in the general population $(>20,000)$ based on an elevated risk of T2DM and CVD, as described elsewhere. ${ }^{32}$ Inclusion criteria were Caucasian descent, age $>40$ years, and one or more cardiometabolic risk factors (ie, BMI $>25 \mathrm{~kg} / \mathrm{m}^{2}$, use of antihypertensive medication, positive family history of T2DM, history of gestational diabetes and/or glycosuria). The CODAM study was approved by the Medical Ethics Committee of the Maastricht University Medical Center. All participants gave written informed consent, and the described methods were carried out in accordance with the approved guidelines. Of the 574 participants, 25 had missing data on HDL characteristics, and 16 had missing covariates leaving $\mathrm{n}=533$ for complete case analyses with the EnD Score, CVD, or CVE as outcomes $(\mathrm{NGM} \mathrm{n}=279$, (pre)diabetes $\mathrm{n}=254) . \mathrm{N}=37$ also had missing data on cIMT leaving $\mathrm{n}=496$ (NGM $\mathrm{n}=268$, (pre)diabetes $=228$ ).

All participants were asked to stop their lipid-modifying medication 14 days and all other medications on the day before the measurements ( $>80 \%$ adherence). Blood samples were obtained by venipuncture after an overnight fast, and plasma aliquots were stored at $-80^{\circ} \mathrm{C}$ until use. All participants underwent an oral glucose tolerance test (except those with established T2DM), and were categorized as having normal glucose metabolism (NGM), impaired glucose metabolism (IGM, ie, prediabetes) or T2DM as previously described. ${ }^{32-34}$ Glycated hemoglobin $\left(\mathrm{HbA}_{1 \mathrm{c}}\right)$ was determined as previously described. ${ }^{25}$

\section{Cardiovascular measures}

\section{Carotid intima-media thickness (cIMT)}

cIMT was measured at the left and right common carotid artery $10-20 \mathrm{~mm}$ proximal to the carotid bulb with an ultrasound imaging device as reported previously. ${ }^{35}$

\section{Endothelial dysfunction (EnD) score}

Soluble intercellular adhesion molecule (sICAM-1), soluble vascular cell adhesion molecule (sVCAM-1), and soluble endothelial selectin (sEsel) were measured using ELISA assays in serum or EDTA plasma. The measurements were calibrated after cross-validation as described elsewhere. ${ }^{36}$ Baseline von Willebrand factor (vWF) was measured with an in-house ELISA in citrated plasma as described. ${ }^{37}$

\section{Cardiovascular events (CVE)}

CVE was defined as the occurrence of myocardial infarction (MI), coronary bypass, percutaneous coronary intervention and/or stroke reported by questionnaires, as well as signs of MI on an electrocardiogram (Minnesota codes $1-1$ or $1-2$ ), as reported earlier. ${ }^{32}$

\section{Cardiovascular diseases (CVD)}

Prior CVD was defined by the self-reported history of CVE. CVD additionally included signs of coronary ischemia (Minnesota codes 1-3, 4-1, 4-2, 4-3, 5-1, 5-2, 53 , or 7-1); nontraumatic limb amputation; and/or anklebrachial index $<0.9$. $^{32}$

\section{HDL characteristics}

HDL-C was determined in EDTA plasma using the HDLC plus assay (Roche Diagnostics, Mannheim, Germany), and apoA-I was determined by immunoephelometric assays as reported earlier. ${ }^{32}$ HDL size and HDL-P were measured using nuclear magnetic resonance spectroscopy as described previously (available at www.nightingalehealth.com). ${ }^{38}$

\section{Cholesterol efflux capacity (HDL-CEC)}

The measurement of Cholesterol Efflux Capacity was done exactly, as described elsewhere. ${ }^{24}$ Briefly, HDL-CEC toward apoB-depleted plasma was measured using THP-1 human monocytes that were differentiated into macrophages by the addition of $100 \mathrm{nM}$ phorbol myristate acetate (PMA). ${ }^{39,40}$ Differentiated THP-1 macrophages were then loaded with acetylated LDL $(50 \mu \mathrm{g}$ protein $/ \mathrm{mL})$ and $1 \mu \mathrm{Ci} / \mathrm{ml}{ }^{3} \mathrm{H}$-cholesterol (PerkinElmer, Boston, MA) for 24 hours followed by equilibration for 24 hours in RPMI 1640 medium containing $2 \%$ bovine serum albumin. ${ }^{40}$ After equilibration, efflux towards $2 \%$ apoB-depleted plasma was performed for 5 hours. ApoB-depleted plasma was generated by precipitating apoB-containing lipoproteins using polyethylene glycol (PEG 600, Sigma, St Louis, MO) in 10 mM HEPES ( $\mathrm{pH} 08.0=$ according to a commonly used protocol). ${ }^{16-18,24,41}$ CEC measurements were performed in duplicates and at the same time, using the same reagents to limit potential variations due to different assay conditions. Values for HDL-CEC were normalized using a standard curve of different concentrations of pooled apoBdepleted control plasma to correct for potential interassay variation across plates and expressed in arbitrary units. ${ }^{41}$ The intraassay $\mathrm{CV}$ of the method was previously determined to be $5.4 \%$, the interassay CV $7.9 \% .^{24,41}$

\section{Other covariates}

BMI was calculated as weight $(\mathrm{kg}) /$ height $(\mathrm{m})$ squared. ${ }^{42}$ Waist circumference was measured as before. ${ }^{35}$ Smoking behavior, physical activity, ${ }^{43}$ dietary calorie intake, ${ }^{33}$ use of antihypertensive, glucose-lowering, and lipid-medication $^{42}$ was assessed with on-site administered questionnaires. Blood pressure was measured as described before. ${ }^{24}$ LDL-C was calculated with the Friedewald formula. ${ }^{32}$ Triglycerides (TGs) and apoB were determined as reported earlier. ${ }^{32}$

\section{Statistical analyses}

Categorical variables are presented as frequencies and percentages. Normally distributed continuous variables are 
Table 1 Characteristics of the study participants divided in CEC tertiles

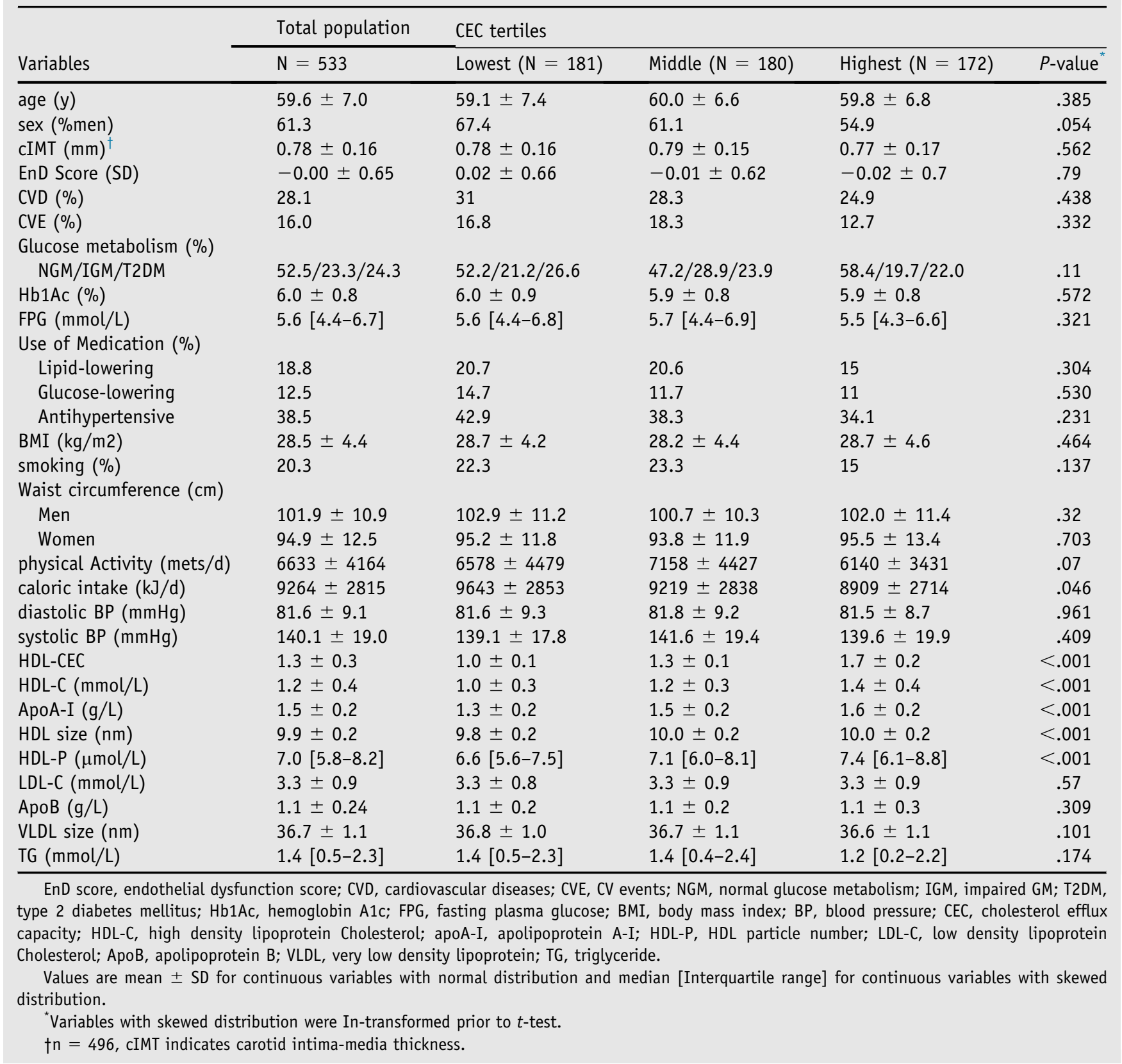

presented as mean \pm standard deviation, skewed variables are presented as median with interquartile range (IQR). Skewed variables (ie, fasting plasma glucose (FPG), HDL$\mathrm{P}$, TG, and EnD markers) were ln-transformed to achieve normal distribution prior to analysis.

For defining an overall measure of endothelial dysfunction, a composite score was calculated using sICAM-1, sVCAM-1, sEsel, and vWF. For this, each individual lntransformed biomarker measurements were first standardized and the individual Z-Scores were averaged into the endothelial dysfunction score (EnD Score). For allowing a direct comparison of the effect sizes for the EnD Score and cIMT, cIMT was also standardized. For the same reason,
HDL characteristics were also converted into their respective Z-Scores.

Cross-sectional associations of HDL characteristics (HDL-CEC, HDL-C, apoA-I, HDL size, HDL-P) with the EnD Score $(\mathrm{N}=533)$ and with cIMT $(\mathrm{N}=496)$ were investigated using linear regression analysis. Logistic regression analyses were done for $\mathrm{CVD}(\mathrm{N}=533,150$ CVD cases) and CVE ( $\mathrm{N}=533,85$ CVE cases). All regression coefficients are presented as crude effect sizes (Model 1) and adjusted for several potential confounders: Model 2: adjusted for age (yrs) and sex, Model 3 (full model): additionally adjusted for smoking (\%), physical activity (mets/ $\mathrm{d})$, caloric intake $(\mathrm{KJ} / \mathrm{d})$, use of glucose-lowering, lipid- 
modifying, and antihypertensive medication (all yes/no), BMI $\left(\mathrm{kg} / \mathrm{m}^{2}\right)$, systolic blood pressure $(\mathrm{mmHg})$, diastolic blood pressure $(\mathrm{mmHg})$, fasting plasma glucose (mmol/ L), IGM (yes/no), T2DM (yes/no).

To determine if the associations differed between individuals with NGM and those with disturbed glucose metabolism (prediabetes or diabetes), we repeated the above-mentioned analyses stratified according to normal or disturbed glucose metabolism. We also performed an interaction analysis by adding interaction terms (HDL characteristic [eg, HDL-CEC] $\times$ glucose metabolism [normal or disturbed]) to the main analyses.

HDL-CEC was significantly higher in women compared to men, but the strength of association of HDL-CEC with cIMT, EnD Score, CVD and CVE did not differ between women and men.

Statistical analyses were performed using IBM SPSS Statistics Version 23. For the main effects $P<.05$, and for interaction terms $P_{\text {interaction }}<.10$ was considered statistically significant.

\section{Results}

\section{The CODAM study population}

The overall characteristics of the CODAM study as a whole and across tertiles of HDL-CEC are shown in Table 1. For the total population, the mean age was $59.6 \pm 7.0$ years, $61.3 \%$ were men, and $24.2 \%$ had T2DM. Mean values for systolic blood pressure were
$140.1 \pm 19.0 \mathrm{mmHg}$, diastolic blood pressure $81.6 \pm 9.1 \mathrm{mmHg}$, and $20 \%$ were current smokers. $28.1 \%$ and $16.0 \%$ were previously diagnosed with CVD and CVE, respectively. Prevalence of normal glucose metabolism, prediabetes, or diabetes, and Hb1Ac levels were not significantly different among HDL-CEC tertiles, while HDL-C, apoA-I, HDL size, and HDL-P were higher in those individuals who were in the highest CEC tertiles. cIMT, EnD, CVD, and CVE did not differ among CEC tertiles.

The associations of HDL-CEC with the atherosclerosis surrogates (ie, cIMT and EnD Score) are shown in Table 2. In crude analyses, cIMT was not associated with HDL-CEC ( $\beta=-0.04$, [95\% CI $-0.13 ; 0.05], P=.413)$, but was significantly and inversely associated with HDL-C, apoAI, and HDL-size, but not with HDL-P. These associations were attenuated and no longer significant in fully adjusted analyses (HDL-C: $\beta=-0.07[-0.16 ; 0.02], P=.130$; apoA-I: $\beta=-0.04[-0.13 ; 0.05] P=.408$; HDL size: $\beta=-0.03[-0.12 ; 0.06] P=.527)$. Likewise, HDLCEC was not associated with the EnD Score, neither crude ( $\beta=-0.01[-0.09 ; 0.08] P=.897)$ nor after adjustment for potential confounders. In contrast, HDL-C, apoA-I, HDL size, and HDL-P, were all significantly and inversely associated with the EnD Score and remained so in the fully adjusted analyses (HDL-C: $\beta=-0.23[-0.31 ;-0.14]$ $P<.001$, apoA-I: $\beta=-0.16[-0.24 ;-0.07] P<.001$, HDL size: $\beta=-0.12[-0.21 ;-0.03], P=.008$, HDLP: $\beta=-0.10[-1.8 ;-0.01], P=.028)$.

Because all the HDL characteristics differed between CEC tertiles (Table 1), we adjusted the analyses of CEC

Table 2 Relationship of HDL-CEC and other HDL characteristics with CIMT and the EnD Score

\begin{tabular}{|c|c|c|c|c|c|c|c|}
\hline \multirow[b]{3}{*}{ HDL-characteristic (Z-Score) } & \multirow[b]{3}{*}{ Model } & \multicolumn{3}{|c|}{ cIMT (Z-Score) } & \multicolumn{3}{|c|}{ EnD (Z-Score) } \\
\hline & & \multicolumn{3}{|l|}{$N=496$} & \multicolumn{3}{|l|}{$N=533$} \\
\hline & & std $\beta$ & $95 \% \mathrm{CI}$ & $P$-value & std $\beta$ & $95 \% \mathrm{CI}$ & $P$-value \\
\hline \multirow[t]{3}{*}{ HDL-CEC } & 1 & -0.037 & $-0.126 ; 0.052$ & .413 & -0.006 & $-0.092 ; 0.080$ & .897 \\
\hline & 2 & -0.027 & $-0.113 ; 0.058$ & .532 & -0.047 & $-0.131 ; 0.037$ & .276 \\
\hline & 3 & -0.022 & $-0.106 ; 0.062$ & .603 & -0.007 & $-0.085 ; 0.071$ & .863 \\
\hline \multirow[t]{3}{*}{ HDL-C } & 1 & -0.127 & $-0.215 ;-0.039$ & .005 & -0.292 & $-0.375 ;-0.209$ & $<.001$ \\
\hline & 2 & -0.090 & $-0.179 ;-0.001$ & .048 & -0.335 & $-0.419 ;-0.252$ & $<.001$ \\
\hline & 3 & -0.071 & $-0.164 ; 0.021$ & .130 & -0.226 & $-0.312 ;-0.140$ & $<.001$ \\
\hline \multirow[t]{3}{*}{ ApoA-I } & 1 & -0.089 & $-0.178 ; 0.001$ & .051 & -0.195 & $-0.280 ;-0.110$ & $<.001$ \\
\hline & 2 & -0.044 & $-0.135 ; 0.048$ & .350 & -0.240 & $-0.327 ;-0.152$ & $<.001$ \\
\hline & 3 & -0.039 & $-0.132 ; 0.054$ & .408 & -0.158 & $-0.244 ;-0.073$ & $<.001$ \\
\hline \multirow[t]{3}{*}{ HDL size } & 1 & -0.093 & $-0.180 ;-0.005$ & .038 & -0.176 & $-0.260 ;-0.091$ & $<.001$ \\
\hline & 2 & -0.054 & $-0.144 ; 0.035$ & .235 & -0.222 & $-0.309 ;-0.134$ & $<.001$ \\
\hline & 3 & -0.030 & $-0.123 ; 0.063$ & .527 & -0.118 & $-0.205 ;-0.031$ & .008 \\
\hline \multirow[t]{3}{*}{ HDL-P } & 1 & -0.076 & $-0.166 ; 0.013$ & .095 & -0.061 & $-0.147 ; 0.026$ & .168 \\
\hline & 2 & -0.021 & $-0.114 ; 0.072$ & .652 & -0.085 & $-0.175 ; 0.006$ & .066 \\
\hline & 3 & -0.060 & $-0.154 ; 0.034$ & .208 & -0.097 & $-0.184 ;-0.011$ & .028 \\
\hline
\end{tabular}

Std $\beta$, standardized regression coefficient.

Linear regression analyses, Model 1: crude, Model 2: adjusted for age, sex, Model 3 additionally adjusted for smoking, BMI, medication usage (antihypertensive, glucose- and lipid-lowering), physical activity, caloric intake, systolic and diastolic blood pressure, fasting plasma glucose, and glucose metabolism state. 


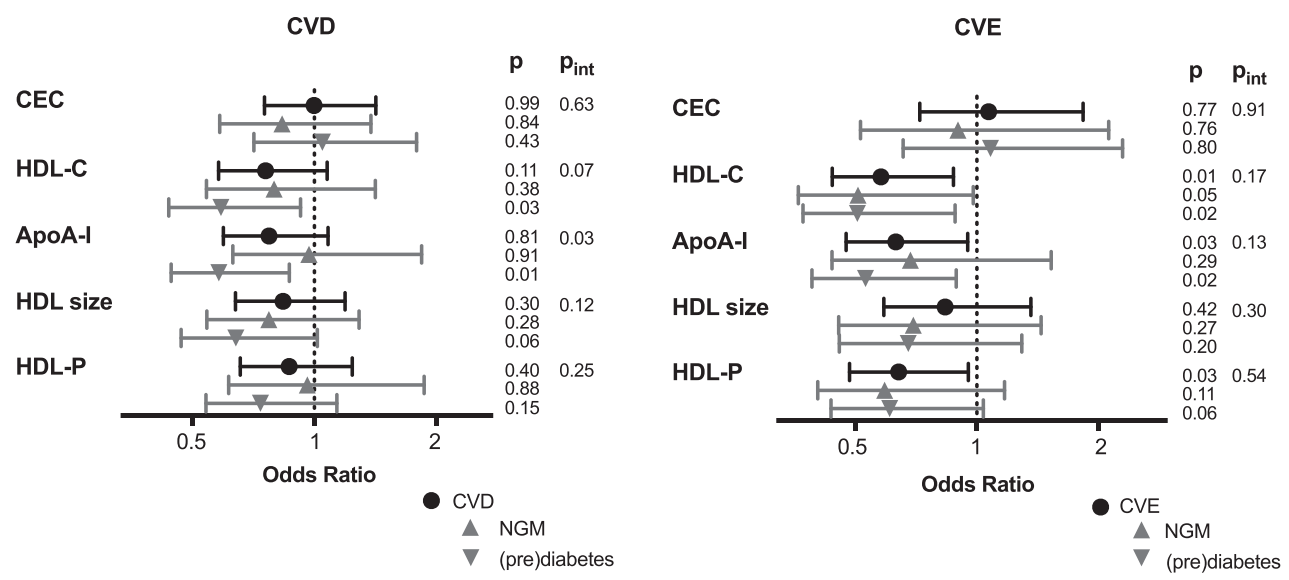

Figure 1 Forrest plot indicating the relationship of HDL characteristics and prevalent cardiovascular diseases (CVD) and CV events (CVE) risk in the whole population, as well as stratified analyses. Logistic regression analyses were adjusted for age, sex, smoking, BMI, medication usage (antihypertensive, glucose- and lipid-lowering), physical activity, caloric intake, systolic and diastolic blood pressure, fasting plasma glucose, and glucose metabolism state. CVD N = 383, 150 CVD cases (NGM: N = 279, 67 CVD cases; (pre)diabetes: $\mathrm{N}=253,83 \mathrm{CVD}$ cases) and CVE $\mathrm{N}=448,85 \mathrm{CVE}$ cases (NGM: $\mathrm{N}=279,39 \mathrm{CVE}$ cases, (pre)diabetes: $\mathrm{N}=254,46 \mathrm{CVE}$ cases).

with cIMT and the EnD Score for HDL-C, apoA-I, HDL size, and HDL-P (Supplemental Table S1). This did not change the lack of association of HDL-CEC with cIMT or the EnD Score. We also evaluated if the associations of the individual HDL-characteristics with the atherosclerosis surrogates were independent of plasma triglyceride (TG) and LDL-C levels (Supplemental Table S2). When the analyses presented in Table 2 were additionally adjusted for TG or LDL-C, the associations between the HDL characteristics and cIMT remained essentially unaltered (Supplemental Table S2). Further, none of the previously observed inverse associations of HDL-C, apoA-I, HDL size, and HDL-P with the EnD Score were affected by additional adjustment for LDL-C. Adjustment for TG did, however, slightly attenuate the associations of HDL-size and HDL-P with the EnD Score (for HDL size to $\beta=-0.08$ $[-0.18 ; 0.02] P=.103$, and for HDL-P to $\beta=-0.08$ $[-0.17 ; 0.01] P=.068)$.

Table 3 Relationship of HDL-CEC and other HDL characteristics with cIMT, stratified for normoglycemia and (pre)diabetes

\begin{tabular}{|c|c|c|c|c|c|c|c|c|}
\hline \multirow{4}{*}{$\begin{array}{l}\text { HDL-characteristic } \\
\text { (Z-Score) }\end{array}$} & \multirow[b]{4}{*}{ Model } & \multicolumn{6}{|c|}{ cIMT (Z-Score) } & \multirow[b]{4}{*}{$P_{\text {int }}$} \\
\hline & & \multicolumn{3}{|l|}{ NGM } & \multicolumn{3}{|c|}{ IGM\&T2DM } & \\
\hline & & \multicolumn{3}{|l|}{$N=268$} & \multicolumn{3}{|l|}{$N=228$} & \\
\hline & & std $\beta$ & $95 \%$ CI & $P$-value & std $\beta$ & $95 \% \mathrm{CI}$ & $P$-value & \\
\hline \multirow[t]{3}{*}{ HDL-CEC } & 1 & 0.062 & $-0.056 ; 0.179$ & .303 & -0.152 & $-0.286 ;-0.017$ & .028 & .019 \\
\hline & 2 & 0.059 & $-0.052 ; 0.170$ & .296 & -0.132 & $-0.265 ; 0.001$ & .051 & .023 \\
\hline & 3 & 0.061 & $-0.044 ; 0.167$ & .255 & -0.129 & $-0.263 ; 0.005$ & .060 & .025 \\
\hline \multirow[t]{3}{*}{ HDL-C } & 1 & -0.057 & $-0.172 ; 0.058$ & .328 & -0.181 & $-0.325 ;-0.038$ & .014 & .184 \\
\hline & 2 & -0.035 & $-0.150 ; 0.079$ & .545 & -0.138 & $-0.287 ; 0.012$ & .071 & .216 \\
\hline & 3 & -0.044 & $-0.158 ; 0.070$ & .446 & -0.138 & $-0.293 ; 0.017$ & .081 & .169 \\
\hline \multirow[t]{3}{*}{ ApoA-I } & 1 & 0.007 & $-0.111 ; 0.125$ & .905 & -0.188 & $-0.325 ;-0.025$ & .007 & .033 \\
\hline & 2 & 0.031 & $-0.088 ; 0.149$ & .611 & -0.132 & $-0.277 ; 0.014$ & .076 & .075 \\
\hline & 3 & 0.011 & $-0.105 ; 0.128$ & .848 & -0.118 & $-0.268 ; 0.031$ & .120 & .067 \\
\hline \multirow[t]{3}{*}{ HDL size } & 1 & -0.088 & $-0.196 ; 0.021$ & .114 & -0.056 & $-0.207 ; 0.095$ & .465 & .737 \\
\hline & 2 & -0.061 & $-0.172 ; 0.050$ & .279 & -0.009 & $-0.165 ; 0.147$ & .908 & .823 \\
\hline & 3 & -0.055 & $-0.167 ; 0.056$ & .329 & -0.006 & $-0.170 ; 0.159$ & .947 & .910 \\
\hline \multirow[t]{3}{*}{ HDL-P } & 1 & -0.007 & $-0.130 ; 0.115$ & .909 & -0.169 & $-0.298 ;-0.039$ & .011 & .061 \\
\hline & 2 & 0.043 & $-0.081 ; 0.167$ & .496 & -0.112 & $-0.253 ; 0.029$ & .118 & .080 \\
\hline & 3 & 0.006 & $-0.115 ; 0.128$ & .919 & -0.132 & $-0.276 ; 0.012$ & .073 & .038 \\
\hline
\end{tabular}

Std $\beta$, standardized regression coefficient.

Linear regression analyses, Model 1: crude, Model 2: adjusted for age, sex, Model 3 additionally adjusted for smoking, BMI, medication usage (antihypertensive, glucose- and lipid-lowering), physical activity, caloric intake, systolic and diastolic blood pressure, fasting plasma glucose, and glucose metabolism state. 
Table 4 Relationship of HDL-CEC and other HDL characteristics with the EnD Score, stratified for normoglycemia and (pre)diabetes

\begin{tabular}{|c|c|c|c|c|c|c|c|c|}
\hline \multirow[b]{3}{*}{ HDL-characteristic (Z-Score) } & \multirow[b]{3}{*}{ Model } & \multicolumn{6}{|c|}{ EnD (Z-Score) } & \multirow[b]{3}{*}{$P_{\text {int }}$} \\
\hline & & \multicolumn{3}{|c|}{$\operatorname{NGM}(N=279)$} & \multicolumn{3}{|c|}{ IGM \& T2DM $(N=254)$} & \\
\hline & & $\operatorname{std} \beta$ & $95 \% \mathrm{CI}$ & $P$-value & std $\beta$ & $95 \% \mathrm{CI}$ & $P$-value & \\
\hline \multirow[t]{3}{*}{ HDL-CEC } & 1 & -0.079 & $-0.186 ; 0.028$ & .146 & 0.039 & $-0.094 ; 0.172$ & .564 & .170 \\
\hline & 2 & -0.105 & $-0.210 ;-0.001$ & .047 & 0.039 & $-0.093 ; 0.171$ & .562 & .099 \\
\hline & 3 & -0.076 & $-0.177 ; 0.025$ & .142 & 0.064 & $-0.060 ; 0.188$ & .312 & .211 \\
\hline \multirow[t]{3}{*}{ HDL-C } & 1 & -0.269 & $-0.370 ;-0.168$ & $<.001$ & -0.242 & $-0.383 ;-0.101$ & .001 & .755 \\
\hline & 2 & -0.330 & $-0.432 ;-0.228$ & $<.001$ & -0.278 & $-0.424 ;-0.132$ & $<.001$ & .695 \\
\hline & 3 & -0.256 & $-0.362 ;-0.150$ & $<.001$ & -0.185 & $-0.330 ;-0.040$ & .013 & .508 \\
\hline \multirow[t]{3}{*}{ ApoA-I } & 1 & -0.216 & $-0.321 ;-0.111$ & $<.001$ & -0.125 & $-0.160 ; 0.010$ & .069 & .290 \\
\hline & 2 & -0.288 & $-0.394 ;-0.181$ & $<.001$ & -0.144 & $-0.287 ;-0.001$ & .049 & .162 \\
\hline & 3 & -0.225 & $-0.333 ;-0.117$ & $<.001$ & -0.071 & $-0.210 ; 0.067$ & .312 & .102 \\
\hline \multirow[t]{3}{*}{ HDL size } & 1 & -0.212 & $-0.310 ;-0.114$ & $<.001$ & -0.047 & $-0.195 ; 0.100$ & .528 & .062 \\
\hline & 2 & -0.277 & $-0.378 ;-0.176$ & $<.001$ & -0.077 & $-0.231 ; 0.078$ & .331 & .068 \\
\hline & 3 & -0.204 & $-0.309 ;-0.099$ & $<.001$ & 0.03 & $-0.119 ; 0.185$ & .668 & .039 \\
\hline \multirow[t]{3}{*}{ HDL-P } & 1 & -0.193 & $-0.304 ;-0.083$ & .001 & 0.038 & $-0.091 ; 0.166$ & .564 & .005 \\
\hline & 2 & -0.252 & $-0.366 ;-0.138$ & $<.001$ & 0.045 & $-0.093 ; 0.182$ & .523 & .002 \\
\hline & 3 & -0.205 & $-0.319 ;-0.092$ & $<.001$ & 0.037 & $-0.095 ; 0.168$ & .582 & .006 \\
\hline
\end{tabular}

Std $\beta$, standardized regression coefficient.

Linear regression analyses, Model 1: crude, Model 2: adjusted for age, sex, Model 3 additionally adjusted for smoking, BMI, medication usage (antihypertensive, glucose- and lipid-lowering), physical activity, caloric intake, systolic and diastolic blood pressure, fasting plasma glucose, and glucose metabolism state.

\section{Association of HDL characteristics with the prevalence of CVD and CVE}

Next, we performed logistic regression analyses with prevalent CVD and CVE as outcomes. HDL-CEC was not associated with CVD or CVE (Fig. 1). Also, none of the other HDL characteristics were associated with CVD (Fig. 1, panel a). On the other hand, HDL-C (OR 0.61 [95\% CI $0.41 ; 0.90] P=.014$ ), apoA-I (OR 0.67 [95\% CI 0.46; 0.96] $P=.030$ ), and HDL-P (OR 0.87 [95\% CI 0.48 ; 0.97] $P=.031$ ) were inversely associated with prevalent CVE, whereas HDL size was not (Fig. 1, panel b). This implies that one standard deviation higher HDL-C, apoA-I, and HDL-P were associated with approximately 1.6-, 1.5- and 1.2-fold fewer CVE cases, respectively. None of these associations were dependent on plasma TG levels or LDL-C (Supplemental Table S3).

\section{Effect of glucose tolerance status on the associations between HDL characteristics and prevalent cardiovascular outcomes}

Interaction analyses suggested that the strength of the association of CEC with cIMT $\left(P_{\text {interaction }}=.025\right)$ and the EnD Score $\left(P_{\text {interaction }}=.103\right)$ might differ between those with NGM and those with (pre)diabetes. Stratified analyses showed that CEC was not significantly associated with cIMT (Table 3) or the EnD Score (Table 4) in either of the subgroups (cIMT: NGM $\beta=0.06[-0.04 ; 0.17]$ $P=.25$, (pre)diabetes $\beta=-0.13[-0.26 ; 0.01]$
$P=.060$; EnD Score: NGM $\beta=-0.08[-0.18 ; 0.03]$ $P=.142$, (pre)diabetes $\beta=0.06 \quad[-0.06 ; 0.19]$, $P=.312$; full model). Potential interaction was also observed for the strength of the association of apoA-I $\left(P_{\text {interaction }}=.067\right)$ and HDL-P $\left(P_{\text {interaction }}=.038\right)$ with cIMT, but no significant associations were observed within the subgroups (NGM or (pre)diabetes, Table 3). The strength of the associations of HDL-size and HDL-P with the EnD Score also appeared to differ between NGM and (pre)diabetes $\left(P_{\text {interaction }}=.039\right.$ and .006 , respectively). HDL-size and HDL-P were inversely associated with the EnD Score in those with NGM, while such association was not observed in individuals with (pre)diabetes (Table 4). Notably, a significant inverse association between HDL-C and the EnD Score was observed in NGM, as in (pre)diabetes $\left(P_{\text {interaction }}=.508\right)$. Additional adjustments for plasma TG and LDL-C levels did not essentially change the strength of these associations (Supplemental Tables S4 and S5).

HDL-CEC was not associated with CVD in either NGM or (pre)diabetes (Fig. 1, panel a). We did observe a difference in the strength of the association of HDL-C and apoAI with CVD ( $P_{\text {interaction }}=.074$ and .034 , respectively). In individuals with NGM $(\mathrm{N}=67 \mathrm{CVD}$ cases) no association of HDL-C or apoA-I with CVD was observed (HDL-C: $\mathrm{OR}=0.84[0.56 ; 1.25] P=.38$, apoA-I: OR $0.98[0.67$; 1.44] $P=.91)$, while in those with (pre)diabetes $(\mathrm{N}=83$ CVD cases), an increase of one unit SD of HDL-C or apoA-I was associated with 1.5-fold fewer cases (HDL-C: $\mathrm{OR}=0.62[0.40 ; 0.94] P=.026$; apoA-I: $\mathrm{OR}=0.61$ 
$[0.41 ; 0.90] P=.012)$. Adjusting for LDL-C did not change these associations, but adjustment for TG attenuated the strength of association between HDL-C and CVD (Supplemental Table 6). No significant interaction with glucose metabolism status was observed for the associations with CVE ( $P_{\text {interaction }}$ between .13 and .98 ), but for completeness the stratified analyses are included in Figure 1, panel b Notably, a significant inverse association between HDL-C and CVE was observed in those with NGM, as well as those with (pre)diabetes $\left(P_{\text {interaction }}=.171\right)$.

\section{Discussion}

In this observational human cohort, we show that HDLCEC is not associated with parameters of atherosclerosis as reflected by cIMT and the EnD Score, nor with prevalent CVD or CVE, independent of glucose metabolism state. In contrast, other HDL characteristics, that is, HDL-C, apoAI, HDL size, and HDL-P, were inversely associated with the EnD Score and with CVE. The inverse association between HDL-C and the EnD Score was observed in subjects with NGM, as well as those with (pre)diabetes, while the inverse associations with apoA-I, HDL size, and HDL-P were found only in individuals with NGM. On the other hand, higher concentrations of HDL-C and apoA-I were associated with less CVD in individuals with (pre)diabetes, but not in those with NGM.

The role of HDL in atherosclerosis has undergone a major reevaluation in the past 5 years, leading to intensified research on HDL-CEC. ${ }^{44}$ Thus far, the results of these efforts are not uniform and the final conclusion on whether and how HDL-CEC impacts atherosclerosis and CVD/ CVE is yet to be made. Some studies showed that an increase in HDL-CEC could be beneficial for atherosclerosis ${ }^{16}$ as well as CVD/CVE, ${ }^{17,18}$ while others reported the opposite. ${ }^{14,15,20}$ In our current evaluation, we did not find any significant associations for HDL-CEC, which is in line with prevalent $^{20,45}$ and incident studies. ${ }^{46} \mathrm{Li}$ et $\mathrm{al}^{20}$ reported that there was no association of HDLCEC with prevalent $\mathrm{CV}$ risk in a cohort with stable angiographically confirmed CVD after adjustment for traditional risk factors, such as age, sex, smoking, diabetes, hypertension, LDL-C, and HDL-C. However, in the same cohort, they unexpectedly observed an association between higher HDL-CEC and increased incident CV events, suggesting a complex relationship between HDL-CEC and vascular disease. The relation of HDL-CEC might differ between atherosclerotic plaque development (=prevalent CVD) and plaque vulnerability-associated phenotypes for example, myocardial infarction (=incident CVD). Further, a recent study showed that the association of cholesterol mass efflux capacity differs between coronary heart disease and stroke. ${ }^{19}$

In contrast to our null findings for HDL-CEC, other HDL characteristics, that is, HDL-C, apoA-I, HDL size, and HDL-P, were inversely associated with the EnD Score, CVD, and CVE. These HDL-characteristics were not associated with cIMT in our current evaluations, although the direction of the nonsignificant associations with cIMT was also inverse.

In the CODAM cohort, as in several other studies, ${ }^{47-49}$ we observed an inverse association of HDL-C with the EnD Score and CVE, which was independent of LDL-C. Despite these robust inverse associations of HDL-C with a risk of atherosclerosis/CVE, the pharmacological elevation of HDL-C has failed to reduce atherosclerosis ${ }^{50}$ or cardiac events. ${ }^{3,4}$ For this reason, the attention has shifted not only from HDL-C to HDL-CEC but also to further alternative indexes of HDL quantity and/or quality, such as apoAI, HDL size, and HDL-P. ${ }^{51}$ As expected, we found inverse associations of apoA-I with atherosclerosis and CVD/CVE. ApoA-I is the main protein component (70\%) of HDL. Several ways of atheroprotective mechanisms for apoA-I have been proposed, and pharmacological interventions targeting apoA-I are in process (recently reviewed in $^{51,52}$ ). Also in line with our results is the current literature on HDL-P, which consistently reports an inverse association of HDL-P with atherosclerosis, ${ }^{53} \mathrm{CVE},{ }^{54}$ future CAD risk, ${ }^{53,55}$ as well as a lower rate of coronary heart disease (CHD) death. ${ }^{56}$ In our current evaluation, the association of HDL-P with the EnD Score and CVE was slightly attenuated after adjustment for TG, thereby remaining significant for CVE, but not for the EnD Score. The inverse association of HDL size with the EnD Score was more strongly attenuated by adjusting for TG. These findings are consistent with the report of Harchaoui et al., ${ }^{55}$ who showed that the relationship between HDL-size and CAD risk was dependent on metabolic parameters, while the relationship of HDL-P with CAD was not.

Overall, HDL is a heterogeneous lipoprotein fraction encompassing particles that markedly differ in size, density, surface charge, and composition. ${ }^{57}$ It exerts several atheroprotective properties, such as antiapoptotic, antioxidant, and nitric oxide promoting effects. However, which atheroprotective property is the most clinically relevant one, and what subfraction of HDL exerts, which property, remains unknown. Notably, the metabolic milieu may affect various properties of HDL. Therefore, we investigated whether the observed associations differed between those with normoglycemia (NGM) and those with (pre)diabetes. The inverse associations of HDL-P and HDL-size with the EnD Score in subjects with NGM were fully absent in those with (pre)diabetes. In contrast, the inverse association for HDL-C was clearly present in both NGM and (pre)diabetes. Our study population displays the typical dyslipidemic phenotype, that is, high TG and low HDL-C, but not the decreased HDL-size and lower HDL-P that has been shown before. ${ }^{58,59}$ This suggests that in those with NGM, the protective effect of a certain number of HDL particles against the development of EnD is better than for that same number of HDL particles in (pre)diabetes. Thus, HDL particle composition, perhaps partly reflected in HDL size, is of 
interest regarding its protective vascular properties. In this context, a possible compromised antioxidative capability of HDL may be of importance. ${ }^{60}$ It has been shown before that HDL is able to reduce cytokine-induced expression of VCAM-1, ICAM-1, and E-Selectin in vitro ${ }^{61}$ and in vivo. ${ }^{62}$ The observation that the inverse associations of HDL size and HDL-P are lost in (pre)diabetes subjects suggests the loss of their antiinflammatory and endothelium-protective properties in people with impaired glucose metabolism and insulin resistance. In contrast to our observations on the EnD Score, the association of higher concentrations of HDL-C and apoA-I with less CVD was only seen in individuals with (pre)diabetes. This was an unexpected observation that, to some extent, may be explained by more careful monitoring of individuals with (pre)diabetes by health care professionals for signs of development of CVD.

The main strength of this study is the relatively large population, the combination of several HDL characteristics, the availability of the EnD Score and cIMT in addition to CVD/CVE, and the approximately equal numbers of individuals with NGM and (pre)diabetes, which allowed us to perform sufficiently numbered stratified analyses. Further, we are the first to report on the relationship of CEC with CVD/CVE in individuals with (pre)diabetes. The interpretation of our current study is limited by the fact that we measured only one functional aspect of HDL function, that is, its CEC, which is thought to reflect a key cardioprotective HDL functionality. We employed a well-validated HDLCEC assay using human THP-1 cell-derived foam cells as cholesterol donor and apoB-depleted plasma as cholesterol acceptor. Significant positive associations of HDL-CEC with other HDL characteristics (HDL-C, apoA-I, HDL size, HDL-P) substantiate its measurement (Supplemental Fig. 1). However, so far, a gold standard to measure HDLCEC in humans does not exist and the optimal method for HDL isolation remains undecided, likely leading to discrepancies between studies. ${ }^{63}$ The Rader Assay, another HDLCEC method using apoB depleted plasma on murine J774 cells, has been shown to correlate with CVD. ${ }^{17}$ Therefore, we remeasured HDL-CEC in a subset of 57 CODAM samples using the Rader Assay and observed a significant correlation with an intraclass correlation coefficient of 0.6 (data not shown). In our opinion, employing human THP1 monocyte-derived macrophage foam cells for HDL-CEC measurements provides a closer approximation of the condition in human atherosclerotic plaques, due to the use of a homologous system (human cells and human apoB-depleted plasma) and the expression of all relevant efflux transporters ATP-binding cassette transporter A1 (ABCA1), ABCG1 and scavenger receptor B1 (SR-B1). ${ }^{41}$

Further, the associations of HDL characteristics with the 2 surrogate markers of atherosclerosis, EnD Score and cIMT, were not always fully concordant. This may partly be due to the fact that they represent different aspects of atherosclerosis and partly to the measurements themselves. cIMT is a widely used noninvasive measure of atherosclerosis $^{64}$ and has been identified as a reliable indicator of generalized atherosclerosis. ${ }^{65}$ However, cIMT measurements are not standardized, ${ }^{66,67}$ and the sonographer, image analyst, as well as random error, can introduce variability. ${ }^{68}$ Therefore, the difference in the association of these parameters with cIMT and the EnD Score may be due to the sensitivity and variability of the cIMT measurements itself. Further, the washout period for lipid-lowering therapy was 14 days and may not have been sufficient to eliminate all their metabolic effects. However, the CODAM study includes participants with pre-existing CVD; thus, 14 days were considered the maximum period for safety reasons. For the extrapolation of our findings, it should be considered that our study includes individuals with at least one cardiometabolic risk factor. Nonetheless, the cohort represents a large part of typical Western populations, namely middle-aged to elderly individuals with a moderately increased risk of cardiometabolic disease. Lastly, the cross-sectional approach of our study hampers a direct causal interpretation of the observed associations.

\section{Conclusion}

In summary, in a cohort of 533 patients with an elevated risk of CVD and T2DM, we observed that HDL-CEC was not associated with atherosclerosis or CVD/CVE, neither in the whole population nor in individuals with (pre)diabetes, while other HDL characteristics showed atheroprotective associations. Notably, the atheroprotective associations of HDL-size and HDL-P are lost in individuals with (pre) diabetes. The fact that, particularly in T2DM, higher apoAI and HDL-C concentrations were related to less CVD in subjects with (pre)diabetes, independent of CVD risk factors, was an unexpected observation and might be related to more intense cardiovascular risk monitoring and awareness of those individuals.

\section{Acknowledgments}

\section{None.}

\section{References}

1. Rosenson RS, Brewer HB Jr, Ansell BJ, et al. Dysfunctional HDL and atherosclerotic cardiovascular disease. Nat Rev Cardiol. 2016;13:48-60.

2. Badimon JJ, Santos-Gallego CG. HDL dysfunction is the answer in the sphinx's riddle? J Am Coll Cardiol. 2015;66:1486-1488.

3. Barter PJ, Caulfield M, Eriksson M, et al. Effects of torcetrapib in patients at high risk for coronary events. $N$ Engl J Med. 2017;357: 2109-2122.

4. HPS2-THRIVE Collaborative Group. Effects of extended-release niacin with laropiprant in high-risk patients. N Engl J Med. 2014; 371:203-212.

5. Glomset JA. The plasma lecithin:cholesterol acyltransferase reaction. J Lipid Res. 1968;9:155-167.

6. Santos-Gallego CG, Badimon JJ, Rosenson RS. Beginning to understand high-density lipoproteins. Endocrinol Metab Clin North Am. 2014;43:913-947.

7. Rader DJ, Alexander ET, Weibel GL, Billheimer J, Rothblat GH. The role of reverse cholesterol transport in animals and humans and relationship to atherosclerosis. J Lipid Res. 2009;50:S189-S194. 
8. Tall AR, Yvan-Charvet L, Terasaka N, Pagler T, Wang N. HDL, ABC Transporters, and cholesterol efflux: implications for the treatment of atherosclerosis. Cell Metab. 2008;7:365-375.

9. Out R, Hoekstra M, Habets K, et al. Combined deletion of macrophage $\mathrm{ABCA} 1$ and $\mathrm{ABCG} 1$ leads to massive lipid accumulation in tissue macrophages and distinct atherosclerosis at relatively low plasma cholesterol levels. Arterioscler Thromb Vasc Biol. 2008:28:258-264.

10. Yvan-Charvet L, Ranalletta M, Wang N, et al. Combined deficiency of $\mathrm{ABCA} 1$ and ABCG1 promotes foam cell accumulation and accelerates atherosclerosis in mice. $J$ Clin Invest. 2007:117:3900-3908.

11. Feig JE, Rong JX, Shamir R, et al. HDL promotes rapid atherosclerosis regression in mice and alters inflammatory properties of plaque monocyte-derived cells. Proc Natl Acad Sci U S A. 2011;108: 7166-7171.

12. Hewing B, Parathath S, Barrett T, et al. Effects of native and myeloperoxidase-modified apolipoprotein A-I on reverse cholesterol transport and atherosclerosis in mice. Arterioscler Thromb Vasc Biol. 2014;34:779-789.

13. Tang C, Houston BA, Storey C, Leboeuf RC. Both STAT3 activation and cholesterol efflux contribute to the anti-inflammatory effect of apoA-I/ABCA1 interaction in macrophages. J Lipid Res. 2016;57: 848-857.

14. Smoak KA, Aloor JJ, Madenspacher J, et al. Myeloid differentiation primary response protein 88 couples reverse cholesterol transport to inflammation. Cell Metab. 2010;11:493-502.

15. van der Vorst EPC, Theodorou K, Wu Y, et al. High-density lipoproteins exert pro-inflammatory effects on macrophages via passive cholesterol depletion and PKC-NF-кB/STAT1-IRF1 signaling. Cell Metab. 2017;25:197-207.

16. Khera AV, Cuchel M, de la Llera-Moya M, et al. Cholesterol efflux capacity, high-density lipoprotein function, and atherosclerosis. $N$ Engl J Med. 2011;364:127-135.

17. Rohatgi A, Khera A, Berry JD, et al. HDL cholesterol efflux capacity and incident cardiovascular events. N Engl J Med. 2014;371: $2383-2393$.

18. Saleheen D, Scott R, Javad S, et al. Association of HDL cholesterol efflux capacity with incident coronary heart disease events: a prospective case-control study. Lancet Diabetes Endocrinol. 2015;3:507-513.

19. Shea S, Stein JH, Jorgensen NW, et al. Cholesterol mass efflux capacity, incident cardiovascular disease, and progression of carotid plaque the multi-ethnic study of atherosclerosis. Arterioscler Thromb Vasc Biol. 2019;39:89-96.

20. Li XM, Tang WH, Mosior MK, et al. Paradoxical association of enhanced cholesterol efflux with increased incident cardiovascular risks. Arterioscler Thromb Vasc Biol. 2013;33:1696-1705.

21. Sarwar N, Gao P, Seshasai SR, et al. Diabetes mellitus, fasting blood glucose concentration, and risk of vascular disease: a collaborative meta-analysis of 102 prospective studies. Lancet. 2010;375: $2215-2222$.

22. Kubota M, Nakanishi S, Hirano M, et al. Relationship between serum cholesterol efflux capacity and glucose intolerance in Japanese-Americans. J Atheroscler Thromb. 2014;21:1087-1097.

23. Apro J, Tietge UJF, Dikkers A, Parini P, Angelin B, Rudling M. Impaired cholesterol efflux capacity of high-density lipoprotein isolated from interstitial fluid in type 2 diabetes mellitus-brief report. Arterioscler Thromb Vasc Biol. 2016;36:787-791.

24. Annema W, Dikkers A, de Boer JF, et al. Impaired HDL cholesterol efflux in metabolic syndrome is unrelated to glucose tolerance status: the CODAM study. Sci Rep. 2016;6:1-9.

25. Low H, Hoang A, Forbes J, et al. Advanced glycation end-products (AGEs) and functionality of reverse cholesterol transport in patients with type 2 diabetes and in mouse models. Diabetologia. 2012;55: 2513-2521.

26. Dullaart RPF, Annema W, de Boer JF, Tietge UJF. Pancreatic $\beta$-cell function relates positively to HDL functionality in well-controlled type 2 diabetes mellitus. Atherosclerosis. 2012;222:567-573.

27. de Vries R, Groen AK, Perton FG, et al. Increased cholesterol efflux from cultured fibroblasts to plasma from hypertriglyceridemic type 2 diabetic patients: roles of pre $\beta$-HDL, phospholipid transfer protein and cholesterol esterification. Atherosclerosis. 2008;196:733-741.

28. Yassine HN, Belopolskaya A, Schall C, Stump CS, Lau SS, Reaven PD. Enhanced cholesterol efflux to HDL through the ABCA1 transporter in hypertriglyceridemia of type 2 diabetes. Metabolism. 2014;63:727-734.

29. Nestel P, Hoang A, Sviridov D, Straznicky N. Cholesterol efflux from macrophages is influenced differentially by plasmas from overweight insulin-sensitive and-resistant subjects. Int J Obes. 2012;36:407-413.

30. Chamaria S, Johnson KW, Vengrenyuk Y, et al. Intracoronary imaging, cholesterol efflux, and transcriptomics after intensive statin treatment in diabetes. Sci Rep. 2017;7:1-13.

31. Pajunen P, Syvänne M, Castro G, Nieminen MS, Taskinen MR. Cholesterol efflux capacity in vitro predicts the severity and extent of coronary artery disease in patients with and without type 2 diabetes. Scand Cardiovasc J. 2001;35:96-100.

32. Jacobs M, van Greevenbroek MM, van der Kallen CJ, et al. Low-grade inflammation can partly explain the association between the metabolic syndrome and either coronary artery disease or severity of peripheral arterial disease: the CODAM study. Eur J Clin Invest. 2009;39: 437-444.

33. Du H, van der A DL, van Bakel MM, et al. Glycemic index and glycemic load in relation to food and nutrient intake and metabolic risk factors in a Dutch population. Am J Clin Nutr. 2008;87:655-661.

34. Deckers JG, Schellevis FG, Fleming DM. WHO diagnostic criteria as a validation tool for the diagnosis of diabetes mellitus: A study in five European countries. Eur J Gen Pract. 2006;12:108-113.

35. Engelen L, Ferreira I, Gaens KH, et al. The association between the374T/A polymorphism of the receptor for advanced glycation endproducts gene and blood pressure and arterial stiffness is modified by glucose metabolism status: the Hoorn and CoDAM studies. $J$ Hypertens. 2010;28:285-293.

36. van Bussel BCT, Ferreira I, van de Waarenburg MPH, et al. Multiple inflammatory biomarker detection in a prospective cohort study: a cross-validation between well-established single-biomarker techniques and an electrochemiluminescense-based multi-array platform. PLoS One. 2013;8:e58576.

37. Stam F, van Guldener C, Schalkwijk CG, ter Wee PM, Donker AJ, Stehouwer CD. Impaired renal function is associated with markers of endothelial dysfunction and increased inflammatory activity. Nephrol Dial Transplant. 2003;18:892-898.

38. Würtz P, Kangas AJ, Soininen P, Lawlor DA, Davey Smith G, Ala-Korpela M. Quantitative serum nuclear magnetic resonance metabolomics in large-scale epidemiology: a primer on -omic technologies. Am J Epidemiol. 2017;186:1084-1096.

39. Kappelle PJWH, de Boer JF, Perton FG, et al. Increased LCAT activity and hyperglycaemia decrease the antioxidative functionality of HDL. Eur J Clin Invest. 2012;42:487-495.

40. Annema W, Nijstad N, Tölle M, et al. Myeloperoxidase and serum amyloid A contribute to impaired in vivo reverse cholesterol transport during the acute phase response but not group IIA secretory phospholipase A(2). J Lipid Res. 2010;51:743-754.

41. Ebtehaj S, Gruppen EG, Bakker SJL, Dullaart RPF, Tietge UJF. HDL (high-density lipoprotein) cholesterol efflux capacity is associated with incident cardiovascular disease in the general population. Arterioscler Thromb Vasc Biol. 2019;39:1874-1883.

42. van Greevenbroek MMJ, Jacobs M, van der Kallen CJH, et al. The cross-sectional association between insulin resistance and circulating complement $\mathrm{C} 3$ is partly explained by plasma alanine aminotransferase, independent of central obesity and general inflammation (the CODAM study). Eur J Clin Invest. 2011;41:372-379.

43. Wendel-Vos GCW, Schuit AJ, Saris WHM, Kromhout D. Reproducibility and relative validity of the short questionnaire to assess health-enhancing physical activity. J Clin Epidemiol. 2003;56: $1163-1169$.

44. Fisher EA, Feig JE, Hewing B, Hazen SL, Smith JD. HDL Function, Dysfunction, and Reverse Cholesterol Transport. Arterioscler Thromb Vasc Biol. 2012;32:2813-2820. 
45. Mutharasan RK, Thaxton CS, Berry J, et al. HDL efflux capacity, HDL particle size, and high-risk carotid atherosclerosis in a cohort of asymptomatic older adults: the Chicago Healthy Aging Study. J Lipid Res. 2017;58:600-606.

46. De Vries R, Groen AK, Dullaart RP. Cholesterol efflux capacity and atherosclerosis. N Engl J Med. 2011;364:1472-1475.

47. Assmann G, Schulte H, Von Eckardstein A, Huang Y. High-density lipoprotein cholesterol as a predictor of coronary heart disease risk. The PROCAM experience and pathophysiological implications for reverse cholesterol transport. Atherosclerosis. 1996;124:S11-S20.

48. Barter P, Gotto AM, LaRosa JC, et al. HDL cholesterol, very low levels of LDL cholesterol, and cardiovascular events. $N$ Engl J Med. 2007;357:1301-1310.

49. Gordon T, Castelli WP, Hjortland MC, Kannel WB, Dawber TR. High density lipoprotein as a protective factor against coronary heart disease. Am J Med. 1977;62:707-714.

50. Nissen SE, Tardif JC, Nicholls SJ, et al. Effect of torcetrapib on the progression of coronary atherosclerosis. $N$ Engl J Med. 2007;356: 1304-1316.

51. Kajani S, Curley S, McGillicuddy FC. Unravelling HDL—looking beyond the cholesterol surface to the quality within. Int J Mol Sci. 2018;19:1-23.

52. Zakiev E, Feng M, Sukhorukov V, Kontush A. HDL-targeting therapeutics: past, present and future. Curr Pharm Des. 2017;23:1207-1215.

53. MacKey RH, Greenland P, Goff DC Jr, Lloyd-Jones D, Sibley CT, Mora S. High-density lipoprotein cholesterol and particle concentrations, carotid atherosclerosis, and coronary events: MESA (multi-ethnic study of atherosclerosis). J Am Coll Cardiol. 2012;60:508-516.

54. Parish S, Offer A, Clarke R, et al. Lipids and lipoproteins and risk of different vascular events in the MRC/BHF heart protection study. Circulation. 2012;125:2469-2478.

55. Harchaoui E. High-density lipoprotein particle size and concentration and coronary risk. Ann Intern Med. 2009;150:84-93.

56. Kuller LH, Grandits G, Cohen JD, Neaton JD, Prineas R. Lipoprotein particles, insulin, adiponectin, C-reactive protein and risk of coronary heart disease among men with metabolic syndrome. Atherosclerosis. 2007; 195:122-128.

57. Rye K-A, Barter PJ. Cardioprotective functions of HDL. J Lipid Res. 2014;55:168-179.
58. Mora S, Otvos JD, Rosenson RS, Pradhan A, Buring JE, Ridker PM. Lipoprotein particle size and concentration by nuclear magnetic resonance and incident type 2 diabetes in women. Diabetes. 2010;59: $1153-1160$

59. Festa A, Williams K, Hanley AJ, et al. Nuclear magnetic resonance lipoprotein abnormalities in prediabetic subjects in the insulin resistance atherosclerosis study. Circulation. 2005;111:3465-3472.

60. Viktorinova A, Jurkovicova I, Fabryova L, et al. Abnormalities in the relationship of paraoxonase 1 with HDL and apolipoprotein A1 and their possible connection to HDL dysfunctionality in type 2 diabetes. Diabetes Res Clin Pract. 2018;140:174-182.

61. Cockerill GW, Rye K-A, Gamble JR, Vadas MA, Barter PJ. High-density lipoproteins inhibit cytokine-induced expression of endothelial cell adhesion molecules. Arterioscler Thromb Vasc Biol. 1995;15: 1987-1994.

62. Dimayuga P, Zhu J, Oguchi S, et al. Reconstituted HDL containing human apolipoprotein A-1 reduces VCAM-1 expression and neointima formation following periadventitial cuff-induced carotid injury in apoE null mice. Biochem Biophys Res Commun. 1999;264:465-468.

63. Triolo M, Annema W, Dullaart RP, Tietge UJ. Assessing the functional properties of high-density lipoproteins: an emerging concept in cardiovascular research. Biomark Med. 2013;7:457-472.

64. Bots ML, Evans GW, Tegeler CH, Meijer R. Carotid intima-media thickness measurements: relations with atherosclerosis, risk of cardiovascular disease and application in randomized controlled trials. Chin Med J (Engl). 2016;129:215-226.

65. Iwakiri T, Yano Y, Sato Y, et al. Usefulness of carotid intima-media thickness measurement as an indicator of generalized atherosclerosis: Findings from autopsy analysis. Atherosclerosis. 2012;225:359-362.

66. Peters SAE, Den Ruijter HM, Bots ML. Ultrasound protocols to measure carotid intima-media thickness: one size does not fit all. J Am Soc Echocardiogr. 2012;25:1135-1137.

67. den Ruijter HM, Peters SA, Anderson TJ, et al. Common carotid intima-media thickness measurements in cardiovascular risk prediction: a meta-analysis. JAMA. 2012;308:796-803.

68. De Groot E, Zwinderman AH, van der Steen AFW, et al. Variance components analysis of carotid and femoral intima-media thickness measurements. Ultrasound Med Biol. 1998;24:825-832. 


\section{Appendix}

Supplemental Table 1 Relationship of HDL-CEC with CIMT \& the EnD Score adjusted for other HDL characteristics

\begin{tabular}{|c|c|c|c|c|c|c|c|}
\hline \multirow[b]{3}{*}{ HDL-characteristic (Z-Score) } & \multirow[b]{3}{*}{ Model } & \multicolumn{3}{|l|}{ cIMT } & \multicolumn{3}{|c|}{ EnD Score } \\
\hline & & \multicolumn{3}{|l|}{$N=496$} & \multicolumn{3}{|l|}{$N=533$} \\
\hline & & std $\beta$ & $95 \% \mathrm{CI}$ & $P$-value & std $\beta$ & $95 \% \mathrm{CI}$ & $P$-value \\
\hline \multirow[t]{5}{*}{ HDL-CEC } & full model & -0.022 & $-0.106 ; 0.062$ & .603 & -0.007 & $-0.085 ; 0.071$ & .863 \\
\hline & full model + HDL-C & 0.002 & $-0.088 ; 0.093$ & .962 & 0.082 & $-0.001 ; 0.164$ & .052 \\
\hline & full model + apoA-I & -0.010 & $-0.101 ; 0.081$ & .827 & 0.057 & $-0.027 ; 0.141$ & .182 \\
\hline & full model + HDL size & -0.015 & $-0.104 ; 0.073$ & .736 & 0.029 & $-0.053 ; 0.111$ & .487 \\
\hline & full model + HDL-P & -0.002 & $-0.093 ; 0.089$ & .970 & 0.033 & $-0.052 ; 0.118$ & .445 \\
\hline
\end{tabular}

Std $\beta$, standardized regression coefficient.

Linear regression analyses, full model adjusted for smoking, BMI, medication usage (antihypertensive, glucose- and lipid-lowering), physical activity, caloric intake, systolic and diastolic blood pressure, fasting plasma glucose, and glucose metabolism state. Additional adjustment for HDL-C, apoA-I, HDL size, and HDL-P was done, respectively.

Supplemental Table 2 Relationship of CEC and other HDL parameters with CIMT \& EnD adjusted for plasma TG Levels and LDL-C

\begin{tabular}{|c|c|c|c|c|c|c|c|}
\hline \multirow[b]{3}{*}{ HDL-characteristic (Z-Score) } & \multirow[b]{3}{*}{ Model } & \multicolumn{3}{|l|}{ CIMT } & \multicolumn{3}{|c|}{ EnD Score } \\
\hline & & \multicolumn{3}{|l|}{$N=496$} & \multicolumn{3}{|l|}{$N=533$} \\
\hline & & std $\beta$ & $95 \% \mathrm{CI}$ & $P$-value & $\operatorname{std} \beta$ & $95 \% \mathrm{CI}$ & $P$-value \\
\hline \multirow[t]{3}{*}{ HDL-CEC } & full model & -0.022 & $-0.106 ; 0.062$ & .603 & -0.007 & $-0.085 ; 0.071$ & .863 \\
\hline & full model + TG & -0.022 & $-0.106 ; 0.062$ & .602 & -0.007 & $-0.085 ; 0.071$ & .858 \\
\hline & full model + LDL-C & -0.022 & $-0.106 ; 0.061$ & .600 & -0.007 & $-0.085 ; 0.072$ & .864 \\
\hline \multirow[t]{3}{*}{ HDL-C } & full model & -0.071 & $-0.164 ; 0.021$ & .130 & -0.226 & $-0.312 ;-0.140$ & $<.0005$ \\
\hline & full model + TG & -0.097 & $-0.200 ; 0.005$ & .064 & -0.218 & $-0.313 ;-0.123$ & $<.0005$ \\
\hline & full model + LDL-C & -0.058 & $-0.152 ; 0.035$ & .221 & -0.231 & $-0.318 ;-0.144$ & $<.0005$ \\
\hline \multirow[t]{3}{*}{ ApoA-I } & full model & -0.039 & $-0.132 ; 0.054$ & .408 & -0.158 & $-0.244 ;-0.073$ & $<.0005$ \\
\hline & full model + TG & -0.044 & $-0.138 ; 0.051$ & .365 & -0.145 & $-0.231 ;-0.058$ & .001 \\
\hline & full model + LDL-C & -0.037 & $-0.129 ; 0.056$ & .436 & -0.158 & $-0.244 ;-0.073$ & $<.0005$ \\
\hline \multirow[t]{3}{*}{ HDL size } & full model & -0.030 & $-0.123 ; 0.063$ & .527 & -0.118 & $-0.205 ;-0.031$ & .008 \\
\hline & full model + TG & -0.052 & $-0.159 ; 0.055$ & .339 & -0.084 & $-0.184 ; 0.017$ & .103 \\
\hline & full model + LDL-C & -0.022 & $-0.115 ; 0.071$ & .638 & -0.119 & $-0.206 ;-0.031$ & .008 \\
\hline \multirow[t]{3}{*}{ HDL-P } & full model & -0.060 & $-0.154 ; 0.034$ & .208 & -0.097 & $-0.184 ;-0.011$ & .028 \\
\hline & full model + TG & -0.065 & $-0.160 ; 0.030$ & .181 & -0.082 & $-0.169 ; 0.006$ & .068 \\
\hline & full model + LDL-C & -0.057 & $-0.151 ; 0.037$ & .231 & -0.097 & $-0.184 ;-0.011$ & .028 \\
\hline
\end{tabular}

Std $\beta$, standardized regression coefficient.

Linear regression analyses, full model adjusted for smoking, BMI, medication usage (antihypertensive, glucose- and lipid-lowering), physical activity, caloric intake, systolic and diastolic blood pressure, fasting plasma glucose, and glucose metabolism state. Additional adjustment for triglycerides (TGs), and LDL-C was done, respectively. 
Supplemental Table 3 Relationship of CEC and other HDL parameters with CVD and CVE adjusted for plasma TG levels and LDL-C

\begin{tabular}{|c|c|c|c|c|c|c|c|}
\hline \multirow[b]{3}{*}{ HDL-characteristic (Z-Score) } & \multirow[b]{3}{*}{ Model } & \multicolumn{3}{|l|}{ CVD } & \multicolumn{3}{|l|}{ CVE } \\
\hline & & \multicolumn{3}{|c|}{$\mathrm{N}=533,150$ CVD cases } & \multicolumn{3}{|c|}{$\mathrm{N}=533,85$ CVE cases } \\
\hline & & OR & 95th CI & $P$-value & OR & 95th CI & $P$-value \\
\hline \multirow[t]{3}{*}{ HDL-CEC } & full model & 0.999 & $0.796 ; 1.253$ & .992 & 1.049 & $0.766 ; 1.437$ & .765 \\
\hline & full model + TG & 0.988 & $0.787 ; 1.240$ & .917 & 1.037 & $0.757 ; 1.419$ & .821 \\
\hline & full model + LDL-C & 1.000 & $0.797 ; 1.255$ & .999 & 2.054 & $0.768 ; 1.445$ & .746 \\
\hline \multirow[t]{3}{*}{ HDL-C } & full model & 0.800 & $0.608 ; 1.053$ & .112 & 0.606 & $0.406 ; 0.904$ & .014 \\
\hline & full model + TG & 0.909 & $0.671 ; 1.230$ & .536 & 0.614 & $0.398 ; 0.947$ & .027 \\
\hline & full model + LDL-C & 0.789 & $0.599 ; 1.040$ & .092 & 0.59 & $0.396 ; 0.878$ & .009 \\
\hline \multirow[t]{3}{*}{ ApoA-I } & full model & 0.814 & $0.627 ; 1.056$ & .814 & 0.667 & $0.462 ; 0.963$ & .030 \\
\hline & full model + TG & 0.845 & $0.649 ; 1.101$ & .213 & 0.676 & $0.467 ; 0.977$ & .037 \\
\hline & full model + LDL-C & 0.814 & $0.628 ; 1.056$ & .122 & 0.674 & $0.467 ; 0.972$ & .035 \\
\hline \multirow[t]{3}{*}{ HDL size } & full model & 0.872 & $0.676 ; 1.126$ & .295 & 0.870 & $0.618 ; 1.223$ & .422 \\
\hline & full model + TG & 1.031 & $0.768 ; 1.383$ & .840 & 0.948 & $0.641 ; 1.402$ & .790 \\
\hline & full model + LDL-C & 0.871 & $0.675 ; 1.124$ & .288 & 0.876 & $0.623 ; 1.231$ & .446 \\
\hline \multirow[t]{3}{*}{ HDL-P } & full model & 0.897 & $0.696 ; 1.156$ & .400 & 0.679 & $0.477 ; 0.965$ & .031 \\
\hline & full model + TG & 0.941 & $0.727 ; 1.217$ & .641 & 0.694 & $0.487 ; 0.991$ & .044 \\
\hline & full model + LDL-C & 0.898 & $0.697 ; 1.158$ & .408 & 0.687 & $0.482 ; 0.979$ & .038 \\
\hline
\end{tabular}

Logistic regression analyses, full model adjusted for smoking, BMI, medication usage (antihypertensive, glucose- and lipid-lowering), physical activity, caloric intake, systolic and diastolic blood pressure, fasting plasma glucose, and glucose metabolism state. Additional adjustment for triglycerides (TGs), and LDL-C was done, respectively.

Supplemental Table 4 Relationship of CEC and other HDL parameter with cIMT, stratified for normoglycemia and (pre)diabetes and adjusted for plasma TG levels and LDL-C

\begin{tabular}{|c|c|c|c|c|c|c|c|c|}
\hline \multirow[b]{4}{*}{ HDL-characteristic (Z-Score) } & \multirow[b]{4}{*}{ Model } & \multicolumn{6}{|l|}{ cIMT } & \multirow[b]{4}{*}{$P_{\text {int }}$} \\
\hline & & \multirow{2}{*}{\multicolumn{3}{|c|}{$\begin{array}{l}\text { NGM } \\
N=268 \\
\end{array}$}} & \multirow{2}{*}{\multicolumn{3}{|c|}{ IGM\&T2DM }} & \\
\hline & & & & & & & & \\
\hline & & std $\beta$ & $95 \% \mathrm{CI}$ & $P$-value & std $\beta$ & $95 \% \mathrm{CI}$ & $P$-value & \\
\hline \multirow[t]{3}{*}{ HDL-CEC } & full model & 0.061 & $-0.044 ; 0.167$ & .255 & -0.129 & $-0.263 ; 0.005$ & .060 & .025 \\
\hline & full model + TG & 0.062 & $-0.044 ; 0.167$ & .249 & -0.130 & $-0.264 ; 0.004$ & .058 & .025 \\
\hline & full model + LDL-C & 0.061 & $-0.044 ; 0.167$ & .252 & -0.130 & $-0.263 ; 0.004$ & .056 & .023 \\
\hline \multirow[t]{3}{*}{ HDL-C } & full model & -0.044 & $-0.158 ; 0.070$ & .446 & -0.138 & $-0.293 ; 0.017$ & .081 & .169 \\
\hline & full model + TG & -0.028 & $-0.151 ; 0.096$ & .659 & -0.239 & $-0.416 ;-0.062$ & .008 & .116 \\
\hline & full model + LDL-C & -0.029 & $-0.146 ; 0.088$ & .625 & -0.134 & $-0.288 ; 0.021$ & .090 & .120 \\
\hline \multirow[t]{3}{*}{ ApoA-I } & full model & 0.011 & $-0.105 ; 0.128$ & .848 & -0.118 & $-0.268 ; 0.031$ & .120 & .067 \\
\hline & full model + TG & 0.019 & $-0.099 ; 0.137$ & .750 & -0.144 & $-0.297 ; 0.009$ & .065 & .063 \\
\hline & full model + LDL-C & 0.018 & $-0.099 ; 0.135$ & .762 & -0.126 & $-0.275 ; 0.023$ & .097 & .047 \\
\hline \multirow[t]{3}{*}{ HDL size } & full model & -0.055 & $-0.167 ; 0.056$ & .329 & -0.006 & $-0.170 ; 0.159$ & .947 & 910 \\
\hline & full model + TG & -0.039 & $-0.166 ; 0.087$ & .540 & -0.079 & $-0.275 ; 0.117$ & .428 & .789 \\
\hline & full model + LDL-C & -0.045 & $-0.158 ; 0.068$ & .433 & -0.017 & $-0.181 ; 0.147$ & .836 & .733 \\
\hline \multirow[t]{3}{*}{ HDL-P } & full model & 0.006 & $-0.115 ; 0.128$ & .919 & -0.132 & $-0.276 ; 0.012$ & .073 & .038 \\
\hline & full model + TG & 0.014 & $-0.108 ; 0.137$ & .818 & -0.151 & $-0.297 ;-0.005$ & .043 & .037 \\
\hline & full model + LDL-C & 0.012 & $-0.109 ; 0.134$ & .841 & -0.135 & $-0.278 ; 0.009$ & .066 & .030 \\
\hline
\end{tabular}

Std $\beta$, standardized regression coefficient.

Linear regression analyses, full model adjusted for smoking, BMI, medication usage (antihypertensive, glucose- and lipid-lowering), physical activity, caloric intake, systolic and diastolic blood pressure, fasting plasma glucose, and glucose metabolism state. Additional adjustment for triglycerides (TGs), and LDL-C was done, respectively. 
Supplemental Table 5 Relationship of CEC and other HDL parameter with EnD, stratified for normoglycemia and (pre)diabetes and adjusted for plasma TG levels and LDL-C

\begin{tabular}{|c|c|c|c|c|c|c|c|c|}
\hline \multirow[b]{4}{*}{ HDL-characteristic (Z-Score) } & \multirow[b]{4}{*}{ Model } & \multicolumn{6}{|c|}{ EnD Score } & \multirow[b]{4}{*}{$P_{\text {int }}$} \\
\hline & & \multicolumn{3}{|l|}{ NGM } & \multicolumn{3}{|c|}{ IGM\&T2DM } & \\
\hline & & \multicolumn{3}{|l|}{$N=279$} & \multicolumn{3}{|l|}{$N=254$} & \\
\hline & & std $\beta$ & $95 \% \mathrm{CI}$ & $P$-value & std $\beta$ & $95 \% \mathrm{CI}$ & $P$-value & \\
\hline \multirow[t]{3}{*}{ HDL-CEC } & full model & -0.076 & $-0.177 ; 0.025$ & .142 & 0.064 & $-0.060 ; 0.188$ & .312 & .103 \\
\hline & full model + TG & -0.074 & $-0.175 ; 0.028$ & .153 & 0.063 & $-0.061 ; 0.187$ & .317 & .109 \\
\hline & full model + LDL-C & -0.076 & $-0.177 ; 0.026$ & .142 & 0.062 & $-0.063 ; 0.186$ & .331 & .103 \\
\hline \multirow[t]{3}{*}{ HDL-C } & full model & -0.256 & $-0.362 ;-0.150$ & $<.0005$ & -0.185 & $-0.330 ;-0.040$ & .013 & .508 \\
\hline & full model + TG & -0.259 & $-0.373 ;-0.144$ & $<.0005$ & -0.151 & $-0.317 ; 0.015$ & .074 & .471 \\
\hline & full model + LDL-C & -0.282 & $-0.390 ;-0.174$ & $<.0005$ & -0.184 & $-0.330 ;-0.039$ & .013 & .459 \\
\hline \multirow[t]{3}{*}{ ApoA-I } & full model & -0.225 & $-0.333 ;-0.117$ & $<.0005$ & -0.071 & $-0.210 ; 0.067$ & .312 & .102 \\
\hline & full model + TG & -0.216 & $-0.325 ;-0.108$ & $<.0005$ & -0.046 & $-0.187 ; 0.095$ & .520 & .085 \\
\hline & full model + LDL-C & -0.231 & $-0.340 ;-0.123$ & $<.0005$ & -0.076 & $-0.216 ; 0.063$ & .281 & .100 \\
\hline \multirow[t]{3}{*}{ HDL size } & full model & -0.204 & $-0.309 ;-0.099$ & $<.0005$ & 0.033 & $-0.119 ; 0.185$ & .668 & .039 \\
\hline & full model + TG & -0.207 & $-0.326 ;-0.088$ & .001 & 0.158 & $-0.020 ; 0.336$ & .081 & .018 \\
\hline & full model + LDL-C & -0.217 & $-0.324 ;-0.111$ & $<.0005$ & 0.028 & $-0.125 ; 0.180$ & .718 & .036 \\
\hline \multirow[t]{3}{*}{ HDL-P } & full model & -0.205 & $-0.319 ;-0.092$ & $<.0005$ & 0.037 & $-0.095 ; 0.168$ & .582 & .006 \\
\hline & full model + TG & -0.195 & $-0.310 ;-0.081$ & .001 & 0.061 & $-0.072 ; 0.194$ & .366 & .005 \\
\hline & full model + LDL-C & -0.210 & $-0.323 ;-0.097$ & $<.0005$ & 0.034 & $-0.098 ; 0.166$ & .611 & .006 \\
\hline
\end{tabular}

Std $\beta$, standardized regression coefficient.

Linear regression analyses, full model adjusted for smoking, BMI, medication usage (antihypertensive, glucose- and lipid-lowering), physical activity, caloric intake, systolic and diastolic blood pressure, fasting plasma glucose, and glucose metabolism state. Additional adjustment for triglycerides (TGs), and LDL-C was done, respectively.

Supplemental Table 6 Relationship of CEC and other HDL parameters with CVD, stratified for normoglycemia and (pre)diabetes and adjusted for plasma TG levels and LDL-C

\begin{tabular}{|c|c|c|c|c|c|c|c|c|}
\hline \multirow[b]{3}{*}{ HDL-characteristic (Z-Score) } & \multirow[b]{3}{*}{ Model } & \multicolumn{6}{|l|}{ CVD } & \multirow[b]{3}{*}{$P_{\text {int }}$} \\
\hline & & \multicolumn{3}{|c|}{$\mathrm{N}=279 ; 67$ CVD cases } & \multicolumn{3}{|c|}{$\mathrm{N}=254 ; 83$ CVD cases } & \\
\hline & & $\mathrm{OR}$ & 95th CI & $P$-value & OR & 95th CI & $P$-value & \\
\hline \multirow[t]{2}{*}{ HDL-CEC } & full model & 0.868 & $0.612 ; 1.232$ & .428 & 1.033 & $0.752 ; 1.419$ & .839 & .625 \\
\hline & full model + TG & 0.927 & $0.557 ; 1.543$ & .771 & 1.022 & $0.673 ; 1.552$ & .920 & .725 \\
\hline \multirow[t]{3}{*}{ HDL-C } & full model & 0.835 & $0.558 ; 1.250$ & .382 & 0.617 & $0.403 ; 0.944$ & .026 & .074 \\
\hline & full model + TG & 0.870 & $0.566 ; 1.339$ & .528 & 0.785 & $0.489 ; 1.259$ & .315 & .110 \\
\hline & full model + LDL-C & 0.800 & $0.530 ; 1.209$ & .290 & 0.617 & $0.403 ; 0.944$ & .026 & .086 \\
\hline \multirow[t]{3}{*}{ ApoA-I } & full model & 0.978 & $0.665 ; 1.440$ & .911 & 0.609 & $0.413 ; 0.898$ & .012 & .034 \\
\hline & full model + TG & 0.993 & $0.674 ; 1.464$ & .973 & 0.639 & $0.428 ; 0.954$ & .029 & .035 \\
\hline & full model + LDL-C & 0.969 & $0.659 ; 1.426$ & .873 & 0.605 & $0.409 ; 0.895$ & .012 & .038 \\
\hline \multirow{2}{*}{ HDL-P } & full model + TG & 0.644 & $0.353 ; 1.175$ & .152 & 0.659 & $0.413 ; 1.054$ & .082 & .222 \\
\hline & full model + LDL-C & 0.778 & $0.555 ; 1.091$ & .145 & 0.968 & $0.648 ; 1.446$ & .874 & .262 \\
\hline
\end{tabular}



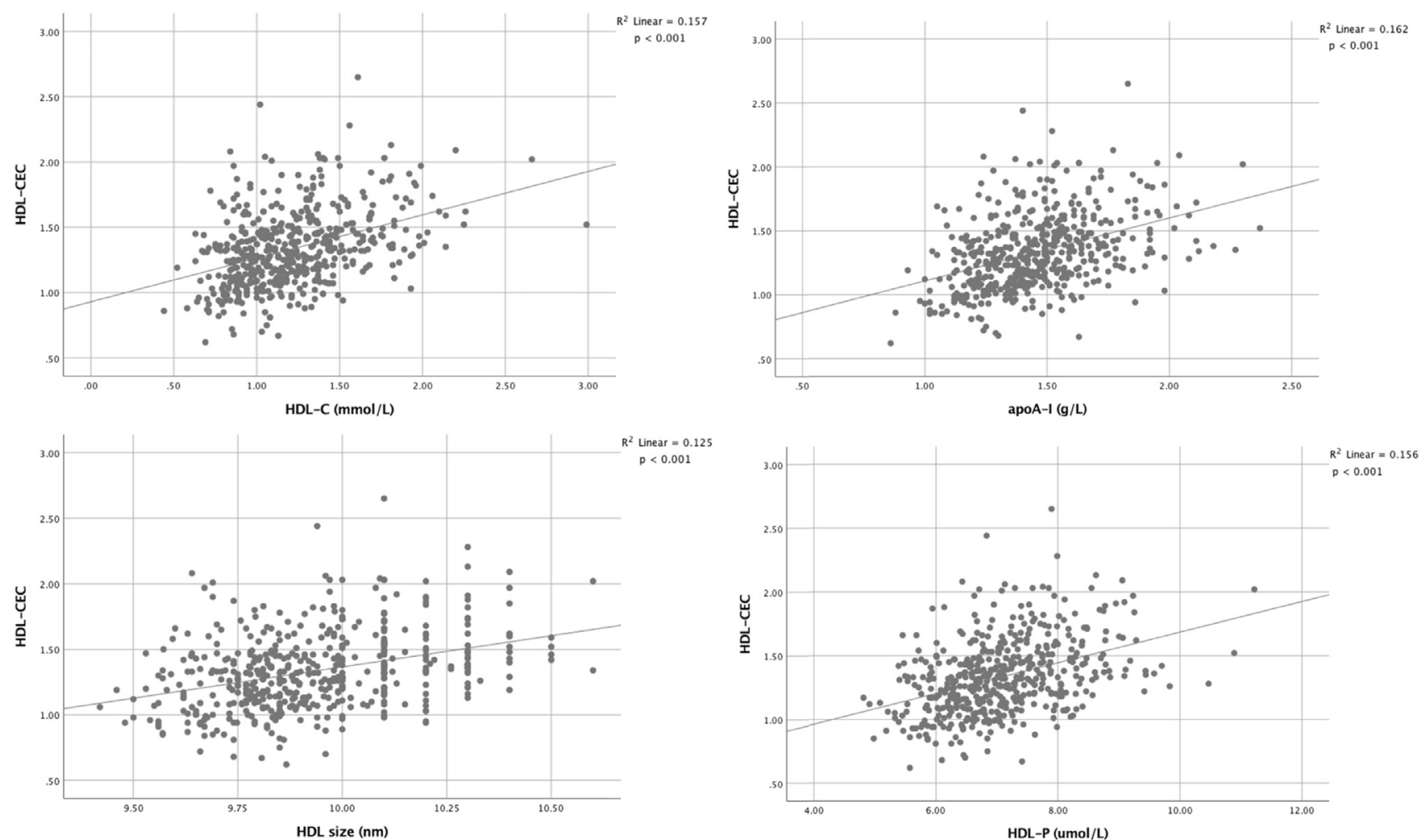

Supplemental Figure 1 Association of HDL-CEC with HDL-C, apoA-I, HDL size, and HDL-P.Scatterplots showing the associations of HDL-CEC with HDL-C (top left), apoA-I (top right), HDL size (bottom left), and HDL-P (bottom right). $\mathrm{N}=533$. 